\title{
Testing acoustic versus physical marking: two complementary methods for individual-based monitoring of elusive species
}

\author{
Paola Laiolo, Matthias Vögeli, David Serrano and José L. Tella \\ P. Laiolo (correspondence), M. Vögeli, D. Serrano and J. L. Tella, Estación Biológica de Doñana (CSIC), Avenida M. Luisa s/n, \\ 41013 Sevilla, Spain. E-mail: paola@ebd.csic.es
}

\begin{abstract}
Individuals of some species differentiate each other on the basis of the acoustic features of their vocalizations, and this can be used in individual-based population monitoring studies. No research has tested for the effectiveness of individual marking through voice recognition as compared to traditional monitoring methods relying on physical marks. We compared voice recognition and physical marking using the Dupont's lark Chersophilus duponti as a study species. This bird needs to be attracted with playback in order to be seen (or captured). We first demonstrated that the territorial calls from a sample of banded males were individually distinctive and constant over time by means of discriminant function analysis, which correctly classified $100 \%$ of marked males. Then, we applied similarity techniques on call spectrotemporal features to define a threshold value of similarity within banded individuals, to be combined with qualitative spectrogram inspection for the classification of all recorded birds. Eventually, we compared the voice and the capture samples, to test for differences in relation to re-location rate, territory fidelity and dispersal movements both within and between years. Voice recognition was less time-consuming than capture-recapture method in the field, but it was useless for monitoring yearlings in call development stage. The two methods provided the same results in terms of territory fidelity and dispersal movements, but differed in re-location rates, which were significantly greater in the case of voice recognition method. By means of physical captures we possibly trapped a large sample of young and silent floaters, with low probability of recapture or recording. This mismatch between methods could bias the estimates of annual survival, which strongly depend on re-location rates. We suggest considering the two methods as complementary rather than alternatives for monitoring populations. Each technique offers unique information, and the two sources should be combined to provide correction factors that would eventually sharpen our knowledge on bird population ecology.
\end{abstract}

It has been shown that vocal individuality can be used as an alternative to physical marking techniques (Terry et al. 2005). Individual marking through voice recognition would permit the identification of individuals on the basis of the spectrotemporal characteristics of the signals they utter. This method can be applied to the study of species that are too elusive to allow mark detection, difficult to catch, or sensitive to the disturbance caused by capture and handling (Terry et al. 2005). The latter point is important both for ethical considerations and methodological reasons. If capture does affect survival or re-capture probabilities (because of capture area avoidance, stress and susceptibility to predators) (De Villiers et al. 1995,
Castelli and Trost 1996), some assumptions of capturemark-recapture approaches are violated, although there are models that incorporate some of these factors in the analysis (Pradel 1993, Kendall and Nichols 1995).

Acoustic techniques of recognition were successfully applied to the study of several bird species, to count effective populations through years (Galeotti and Pavan 1991, Terry and McGregor 2002), to estimate sitefidelity (Eakle et al. 1989, Galeotti and Sacchi 2001) and annual survival (Gilbert et al. 2002), although reidentification could be difficult if acoustic constancy over years is not demonstrated (Puglisi and Adamo 2004). 
In spite of the increasing number of studies on vocal individuality and their potential application for population monitoring, the results obtained with voice recognition were rarely compared with those obtained with traditional markers, and the efficacy of voice recognition was never explicitly tested (Terry et al. 2005). Moreover, past studies were based on rather low sample sizes, sometimes less than 30 individuals. In this paper, we focus on a large sample of individual recordings and captures of Dupont's lark Chersophilus duponti, a species that has been the object of extensive recordings and physical marking in 2004 and 2005. The species is elusive, rare and of conservation concern, and any information on its baseline ecology is difficult to obtain (Garza et al. 2003, Tella et al. 2005). Dupont's lark is also extremely elusive, and need to be attracted with a playback equipment in order to be spotted. This technique has been shown to have negative side-effects on the behaviour of some bird species (avoidance of disturbance areas, behavioural changes and influence on the sampling protocols; Legare et al. 1999). The comparison of data obtained by vocal recognition through recordings and by sightings through playback may serve to tests for the effectiveness of both methodologies.

In this paper, we first demonstrate that the territorial calls of Dupont's lark are individually distinctive and that their acoustic features are constant over time, two requirements for vocal individuality studies. Then, we compare voice recognition and capture-recapture results in relation to re-location rate, territory fidelity, and dispersal movements. We eventually discuss the potential alternative or complementary information provided by the two methods, their limits and the different sampling effort they demand.

\section{Methods}

\section{Study area and species}

The study was carried out in Ebro Valley (Northeastern Spain), a wide dry plain mainly characterised by prevailing cereal cultivations and patches of natural shrub-steppe vegetation of variable size. The study area covers approximately $10,500 \mathrm{~km}^{2}$. The Ebro Valley is the second stronghold for Dupont's lark in Spain, in spite of hosting less than 400 male territories (Tella et al. 2005).

The Dupont's lark is a small, sedentary and territorial passerine that only inhabits flat shrub-steppe habitats of Spain and North Africa (Cramp 1988, Garza and Suárez 1990, Seoane et al. 2006). The species is classified as Endangered in the Spanish Red List (Garza et al. 2004) and Nearly Threatened in the
IUCN Red List (2005). Because of its cryptic colour, secretive behaviour and reluctance to fly, this lark is difficult to observe, and most contacts are auditory. The song, the territorial call, the warning call, the distress call and the alarm whistles are the main vocalisations emitted by males (Laiolo and Tella 2005, Laiolo et al. 2005, Laiolo and Tella 2006, authors unpubl. data). Birds sing mostly in spring, whereas the calls are audible also in autumn. Vocal activities are concentrated in two daily peaks: $1-0.5 \mathrm{~h}$ before dawn (the main peak) and shortly after sunset. Song and territorial call are longrange vocalisations: calling males can be heard at a distance of $1,500 \mathrm{~m}$.

\section{Trapping and banding}

Trapping and observations of banded birds were performed in June-July and September-October 2004, and in March-June and September-October 2005.

Territorial birds were located aurally during surveys carried out before dawn, and trap groups (3-4 spring loaded traps baited with yellow mealworms) were later placed in each localised territory. A playback equipment (CD player and loudspeaker) broadcasting the species song was used to attract birds. Captured birds were banded with a metal ring and a combination of three colour rings to provide individual recognition at distance. They were measured following standardized protocols and a drop of blood was extracted for molecular sexing; eventually, they were released at the capture sites. Playback was later used in the same territories to attract birds and allow re-captures and re-sightings of colour banded individuals. $11 \%$ of 'reencountered' birds was re-captured physically, and the remaining $89 \%$ was re-sighted at the playback area. The species is so elusive that the detection of a representative sample of marked individuals is very unlikely without the help of the playback equipment. We determined the position of all birds captured, recaptured and re-sighted with a Global Positioning System Garmin eTrex Navigator.

For the purpose of this study, we only used male data ( $n=201$ birds), which constitute $76 \%$ of the total number of captured birds in 2004-2005 $(n=265)$. Sampled males include both first-year males after summer moult and older males, which cannot be differentiated on morpho-anatomical basis (both yearlings and adults undergo complete moult in JulySeptember, see Svensson 1992). To facilitate reading, both 're-captures' and 're-sightings' were labelled as 're-captures' and considered as part of the 'capturerecapture method'. 


\section{Recordings and sound measurements}

We focused on the territorial call, which is characterised by high spectrotemporal repeatability within individuals (Laiolo and Tella 2006). Recordings were obtained in male territories in March-April and October 2004, and in March-May and September-October 2005.

A transect was walked in each steppe patch, and birds calling were approached to obtain good quality recordings $($ B30 m). We used a Sony TC-D8 DAT recorder and a Sennheiser ME67 microphone (frequency response 50-20 $000 \mathrm{~Hz}$ ). Bird position during calling was also established by means of a GPS Garmin eTrex Navigator.

To demonstrate that territorial calls can be used for individual identification (i.e. that vocal features remain constant over time), we concentrated on multiplerecordings of banded birds. In different days we repeated playback sessions during daylight to attract, identify and possibly record the vocal response of territorial birds. This technique can allow the simultaneous recording and identification of birds, which are otherwise difficult to observe while calling at night or hidden in the vegetation, although not all birds respond vocally to playback. No differences were found in the spectrotemporal features of marked bird calls recorded in their territories at night and those subsequently elicited by playback after sunrise and in the proximity of the observer. Only call rate became more variable (pers. obs.).

Five males were first captured and recorded in 2004 and then re-captured and recorded in 2005 (mean interval between recordings of the same bird: $260 \mathrm{~d}$, range 158-355), whereas 20 individuals were recorded and identified twice in 2005 (mean time between recordings: $27 \mathrm{~d}$, range 1-126); one male was recorded both between and within years, thus a total of 24 ringed birds was recorded at least twice between September 2004 and October 2005. Another 12 banded birds were identified and immediately recorded calling, but we were unable to record or capture them twice; these individuals were included in the discriminant function analysis (see below).

Sound analyses were carried out with Avisoft SASLab Pro (Version 3.91; Specht 2003), performing a Fast Fourier Transform (sampling frequency 22,050 $\mathrm{Hz}$, FFT length 512, time resolution $8.9 \mathrm{~ms}$, frequency resolution $43 \mathrm{~Hz}$, Window Function: Bartlett). The characteristics of the territorial call of Dupont's lark are described in Laiolo and Tella (2006); for this study, we focused on a greater number of spectro-temporal variables and classified calls at a finer detail than for the above paper. The six 'broad types' of territorial calls described in Laiolo and Tella (2006) were further divided on the basis of note number, totalling 10 finetypes of calls (Fig. 1). Acoustic characteristics were measured in the time domain on envelopes and in frequency domain on fast Fourier transform mean spectra. In each call, we measured overall call duration, the duration and the frequency of each note; when partly or entirely frequency-modulated (i.e. when frequency changed over time) or harsh notes occurred, we also measured the duration, the maximum and minimum frequency of the modulated or harsh portion, and the number of modulations. The number of spectrotemporal variables measured varied from 4 in the simplest call type (1 note) to 17 in the longest call (5 notes; in Fig. 2 an example is given).

An individual can utter up to three types of territorial calls, and the different types are alternated regularly during calling (Laiolo and Tella 2006); individuals uttering 1,2 or 3 call types may all be represented in a population. We define a complete 'call train' as the utterance of the complete repertoire of an individual (1-3 calls). Overall, measurements were taken on 1,612 call trains, totalling 3,138 single calls. Taking into account inter-individual variability in call type number, we built a matrix in which individual identity was listed in rows, whereas the acoustic variables of all the call types in a train were arranged in columns, with 0 -values entered for spectro-temporal variables of call types that were not uttered by an individual (individual $>$ acoustic variables matrix). Therefore, the number of columns in the matrix (111) represented the whole set of variables measured in the 10 call types.

The insertion of 0 -values for the spectrotemporal variables of calls that were not included in an individual's repertoire permitted to generate a single matrix for all data and individuals. In doing this, dissimilarities between birds that did not share any call types were represented by fictitious maximum values. These values were however lower than those actually expected between individuals that did not share any calls (it should rather approach infinite), thus the method may be even conservative. As our aim was to prove acoustic constancy and test for similarities between the same call types and individuals, the fact that maximum distances may be biased should not have conditioned our results.

\section{Quantitative sound analysis}

Two multivariate analyses are commonly applied to vocal individuality studies: discriminant function analysis (discrimination obtained by finding differences) and similarity techniques (grouping data on the basis of similarities among samples) (Gilbert et al. 2002, Terry et al. 2005). As most types of discriminant function analysis can only assign membership if all individuals are known, i.e., they can only locate new recordings to 

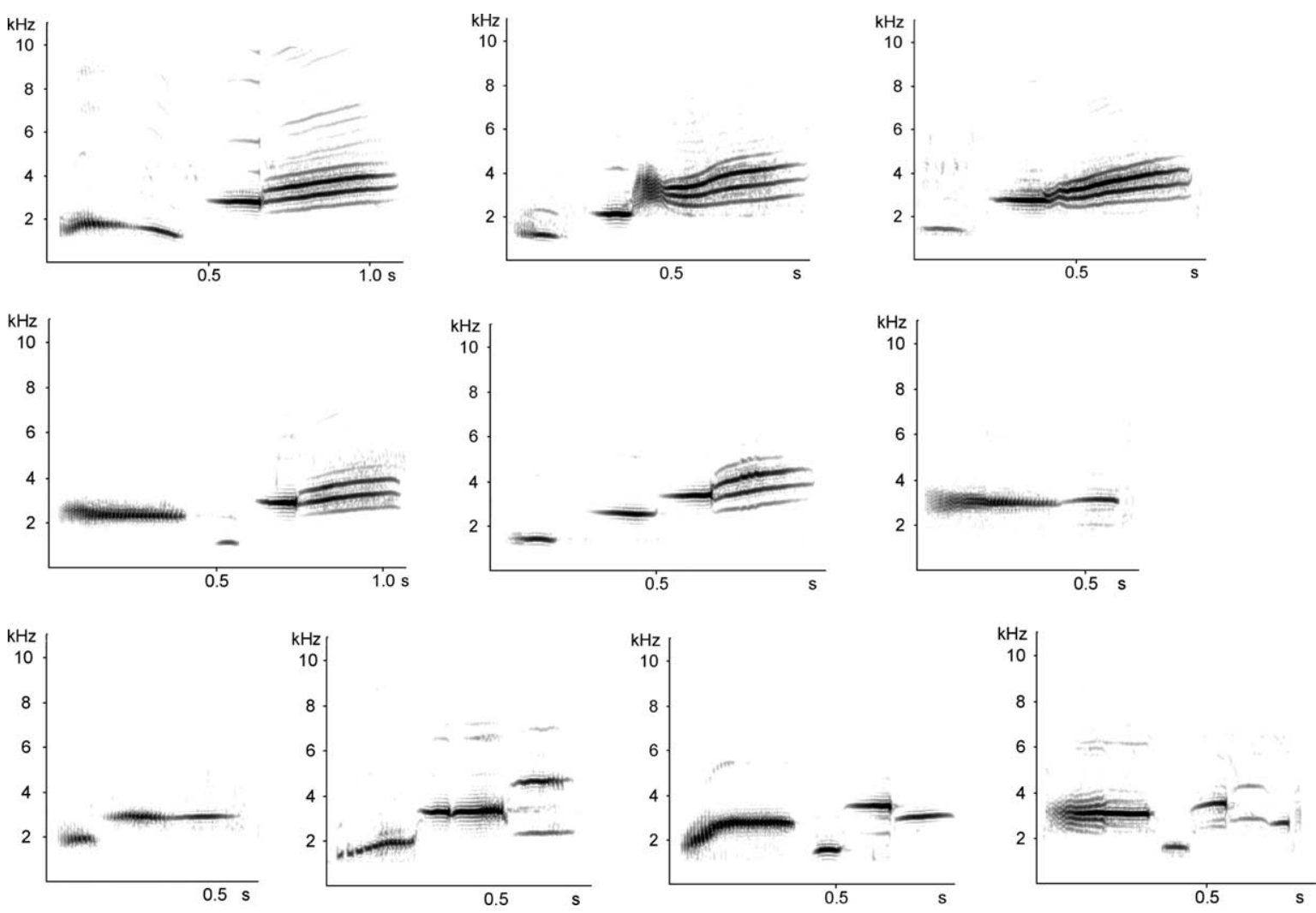

Fig. 1. Spectrograms of the territorial calls uttered by different Dupont's lark males in Ebro Valley.

birds already in the sample, we only used this analysis to test for the discriminant power of the acoustic features of the 36 recorded birds whose identity was known (see above).

Using this sample, we then applied similarity techniques to define threshold values of similarity within individuals, to be used on the whole data set for classification purposes. If a new recorded bird fell outside the within-individual threshold for all birds, it was classified as a new individual (Peake et al. 1998, Terry et al. 2005).

\section{Discrimination and identification of marked birds} We performed discriminant function analysis on standardized data (zero mean and unit standard deviation). We entered in the analysis 401 call trains (from the 36 individuals) and 100 variables; the number of variables was lower than previously stated for the whole data set (111) because some calls were not represented in the repertoire of marked individuals. Prior probability of individual membership was selected so that any given call train was equally likely to be classified to any individual bird (prior $\mathrm{P}=0.028$ ).
To measure similarity between call trains we calculated the Euclidean distances between the acoustic features of pairs of birds (dyads, in keeping with Peake et al. 1998 and Gilbert et al. 2002). Relying on the high stereotypy of call trains uttered in the same day by an individual (Laiolo and Tella, 2006), we calculated the daily mean of each measurement for each bird. Therefore, the sample size for each individual corresponds to the number of days in which it was recorded, not to the total number of recorded call trains. From the pairwise matrix, we calculated acoustic distances for dyads of known: (a) same bird-same year, (b) same bird-different year, (c) different bird-same territory, (d) different birdsame steppe patch, and (e) different bird-different steppe patch. Acoustic distances between birds that turned over in the same territory or occurred in the same patch were calculated to test whether acoustic mimicry between close birds occurred, behaviour found in the complex song of the species (Laiolo and Tella 2005). In order to increase sample size of 'different bird-same territory' dyads, we entered in the analysis 4 cases in which an unmarked bird replaced previously marked bird. These unmarked birds were treated as 4 different individuals, as they were recorded in patches 


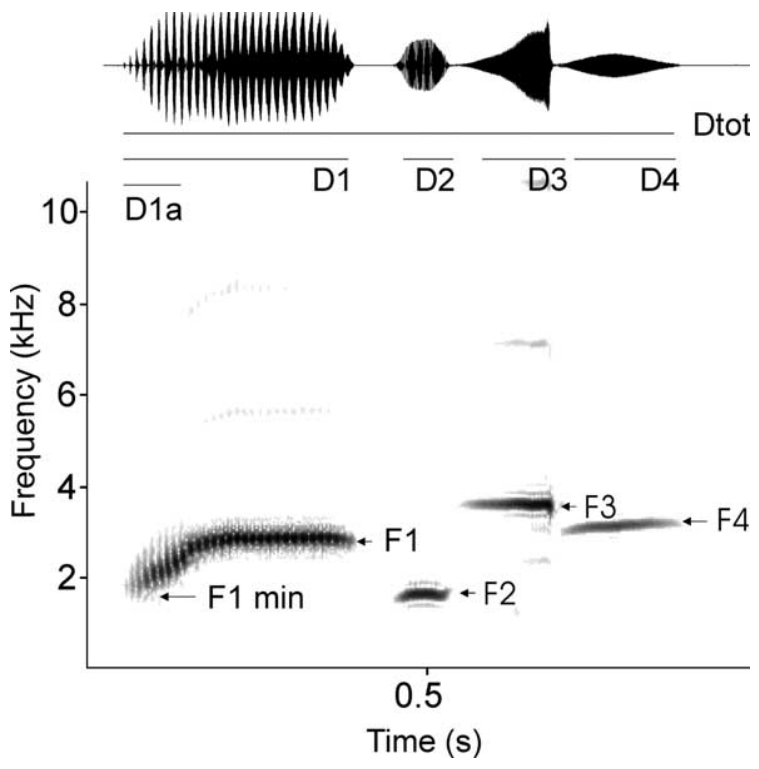

Fig. 2. Example of the acoustic parameters measured in a Dupont's lark call type. Waveform (top) and spectrogram (bottom) are shown. The duration of the whole call (Dtot), of each note (D1, D2, D3, D4) and of the ascending part (in pitch) of the first note (D1a) were measured in the time domain on the envelope. The frequencies of each note (F1, F2, F3, F4) and the minimum frequency of note 1 (F1 min) were measured in the frequency domain on fast Fourier transform mean spectra.

10-45 km far from each other in a short time interval (late spring 2005). The maximum acoustic distance within the same individual was used as a threshold value of similarity for identifying unknown birds.

A Kruskal Wallis test was used to test for differences in acoustic distances among the 5 dyad groups. In order to avoid pseudoreplication derived from comparing a large set of pair-wise distances, we entered in the analysis mean acoustic distance per bird in the case of multiple recordings of the same individual within one year, and mean acoustic distance per steppe patch in the case of different bird-same steppe patch and different bird-different steppe patch dyads. Hence, we performed a Kruskal Wallis test entering 21 same bird-same year acoustic distances, 5 same bird-different year distances, 7 different bird-same territory distances, 12 different bird-same steppe patch distances (i.e. mean interindividual distances for 12 steppe patches), 18 different bird-different steppe patch distances (i.e. mean interindividual distances for 18 steppe patches).

Identification of the whole sample

We used both qualitative and quantitative analysis to identify recorded birds. An archive of spectrograms of call trains was built for each recorded individual, to allow the assessment of similarity through visual inspection of spectrograms (qualitative analysis). This analysis was carried out at blind comparing call trains of all the recorded individuals.

Quantitatively, we calculated Euclidean distances between mean call trains of every pair of recorded birds, and then accepted that individuals recorded in different days were the same if their acoustic distance was less than the maximum known same-bird distance (threshold value), either within or between years (Peake et al. 1998). Discriminant function analysis and Euclidean distance estimations were performed with SPSS (version 13).

\section{Comparisons between voice recognition and capture-recapture methods: re-location probability and movements}

We first tested whether the two techniques showed differences in the probability of male re-location, controlling for the potential confounding effect of the time passed between sampling periods. We considered the following time periods $\left(\mathrm{t}_{1}-\mathrm{t}_{2}\right)$ : within spring (same year), within autumn (same year), from spring to autumn (same year), from spring 2004 to spring 2005, from spring 2004 to autumn 2005, from autumn 2004 to spring 2005, from autumn 2004 to autumn 2005. We performed a logistic regression models with a binomial distribution of errors and a logistic link function. The denominator was the overall number of males located in $t_{1}$ (new captured/recorded males plus those re-captured or already recorded from previous sessions), and the numerator the total number of these males that were re-located in $t_{2}$. We entered two fixed factors and their interaction as predictors: method (capture-recapture or voice recognition) and period (the 7 categories listed above). As we did not detect dispersal events among steppe patches, steppe identity was entered as a random factor.

We tested for differences between periods and methods in dispersal distances using a generalized linear mixed model with a Poisson distribution of errors and a log link function, as data were not normally distributed. Alternatively, we excluded from the analysis birds that did not move (distance $=0$ ), in order to perform the above model with a Normal distribution of errors and an identity link function on $\log (\mathrm{x}+0.5)$ transformed data (Pasinelli et al. 2004). Individual and steppe identity were entered as random factors.

We also tested whether the two techniques differently highlighted territory switch, i.e. if they detected different proportions of territory changes within fragments. We first calculated the mean nearest neighbour distance between territories for each steppe patch (territory-switch threshold), and then classified territory 
changes as movements longer than the mean territoryswitch threshold for that patch. Hence, we performed a logistic regression with a binomial distribution of errors in which the dependent variable was binomial (0: the territory was maintained, 1: the territory changed). As independent variables, we entered period and technique (fixed factors); steppe identity was entered as random factor. All the above generalized linear mixed models were performed with the macro GLIMMIX of SAS (version 8e).

\section{Results}

\section{Acoustic discrimination and individualisation of banded birds}

Discriminant function analysis on 100 acoustic variables correctly classified $100 \%$ of call trains to the individual from which they were recorded, either recorded within the same day, in different days, seasons or years. The first 5 discriminant functions explained 94\% of overall data variation, and had eigenvalues $>22.4$, Wilks' Lambda values $=0.0$ and $\mathrm{x}^{2}$ values $>61,739$ (P B0.001).

Acoustic (Euclidean) distances were calculated on a pairwise matrix of the 36 ringed individuals plus the four unmarked individuals that replaced banded birds in their territories. The maximum value of known same-bird acoustic distance (either within or between years) was 1.0, whereas the lowest acoustic distance between dyads of different individuals was 1.7, consequently no overlap occurred in the acoustic distances of same bird and different bird dyads.

Significant differences resulted between acoustic distances for dyads of: 1) known same bird-same year, 2) same bird-different year, 3) different bird-same territory, 4) different bird-same steppe patch, and 5) different bird-different steppe patch (Kruskal Wallis test, $\mathrm{x}^{2}=50.6$, $\mathrm{df}=4, \mathrm{~PB} 0.001$ ), and mean acoustic distances within individuals were B0.5 and among individuals $>10$. No significant difference resulted between distances of the same birds recorded within the same or in successive years (Mann Whitney $\mathrm{U}$ test, $\mathrm{U}=$ $34, \mathrm{P}=0.22$ )

\section{Identification of the whole sample of recordings}

Visual inspection of spectrograms of all recorded males led to the identification of 174 different individuals. Quantitative analysis was performed by first calculating acoustic Euclidean distances between recorded individuals, and then accepting that they were the same if their acoustic distance was lower than 1.0, i.e. the acoustic threshold of similarity calculated above. This analysis almost completely confirmed qualitative inspections, but five incorrect matches occurred, thus only 169 individuals were statistically identified. Two of these incorrect matches corresponded to males recorded 35 and $120 \mathrm{~km}$ distant from each other in the same breeding season, whereas the remaining three dyads corresponded to birds recorded in the same patch and days but in different territories. The acoustic distances between these dyads fell below the threshold, but they were significantly greater than those within individuals from these territories in different days (range of acoustic distances for dyads in different territories: $0.53-0.88$; birds in the same territory: 0.18-0.41; Wilcoxon matched pair test, $\mathrm{Z}=1.79$, $\mathrm{n}=5, \mathrm{P}=0.043$ ). Moreover, Mann Whitney $\mathrm{U}$ tests showed that these dyads significantly differed in 58$88 \%$ of their acoustic variables, in spite the same call types being uttered. These five cases were eventually considered as incorrect matches, and a sample of 174 recorded individuals was recognized. In doing this, we also took into account Gilbert et al.'s (2002) criterion of incorporating knowledge on site fidelity to similarity measures, which should be attained by removing matches within the same territory from subsequent matching.

\section{Comparisons between acoustic marking and capture-recapture methods}

In the sample of 174 recorded males, 68 were recorded in 2004 and 106 new individuals were added in 2005. Thirty seven individuals were recorded both in 2004 and in 2005. A total of 201 males were captured in the period 2004-2005, 100 in the first year and 101 in the second year. Overall, 33 birds captured in 2004 were re-sighted or re-captured in 2005. Inter-annual re-location rate was significantly greater for acoustic marking data (54 versus $33 \%, \mathrm{x}^{2}$ tests of independence $=10.5, \mathrm{P}=0.001$ ).

A generalised mixed model showed that both techniques and time periods differed in proportion of re-locations (method: $\mathrm{F}_{1,118}=18.3$, P B 0.001; period: $\mathrm{F}_{6,118}=5.4$, P B 0.001). The interaction between period and method was not significant $\left(\mathrm{F}_{6,118}=0.99, \mathrm{P}=\right.$ 0.43 ). Re-captures tended to be particularly scarce in autumn (Fig. 3).

When considering bird movements, significant differences resulted among periods only when 0-distances were included $\left(\mathrm{F}_{6,79}=2.8, \quad \mathrm{P} \mathbf{B} 0.05\right)$, whereas the model was not significant excluding 0 -values $\left(\mathrm{F}_{6,49}=\right.$ $1.4, \mathrm{P}=0.23$ ). Capture-recapture methods highlighted displacement distances that were not significantly different from those shown by acoustic marking both including or excluding 0-distances $\left(\mathrm{F}_{1,79}=0.7\right.$ and $\mathrm{F}_{1,49}=0.4$ respectively, all $\mathrm{P}>0.40$ ). The median dispersal distance recorded was 113 m (maximum 


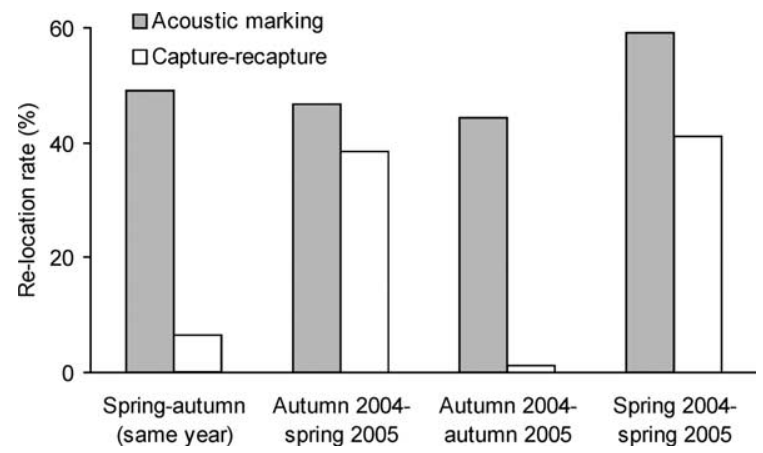

Fig. 3. Percent re-location rate as resulted from acoustic marking and capture-recapture methods. Rates between period $t_{1}$ and $t_{2}$ are calculated as the proportion of birds captured or recorded in $t_{1}$ that were re-located in $t_{2}$.

2,193 $\mathrm{m}$ ) in the case of acoustic marking, and $75 \mathrm{~m}$ (maximum 2,183) with capture-recapture method (Fig. 4). The two methods highlighted movements that tended to be both below or above the mean nearest neighbour distance, the only exception being between autumn 2004 and spring 2005 (Fig. 5). The above results were confirmed by the lack of significant differences in territory fidelity parameter (proportion of territory switches, acoustic marking: $25 \%$; capturerecapture: $21 \%, \mathrm{~F}_{1,78}=0, \mathrm{P}=1$ ). Territory fidelity varied with period $\left(\mathrm{F}_{6,78}=2.24, \mathrm{P}=0.048\right)$, whereas the interaction term between period and method was not significant $\left(\mathrm{F}_{6,78}=1.31, \mathrm{P}=0.26\right)$.

Overall, 182 person days (1,730 h) were spent for trapping and banding activities, whereas 114 person days (724 h) were spent in the field for sound recording, and another 80 full days were spent for sound analysis. Monthly effort was significantly greater for captures than for recordings only when considering the number of hours $(\mathrm{t}=2.0, \mathrm{P}=0.03)$, whereas no differences resulted in the days of fieldwork $(t=0.4$, $\mathrm{P}=0.34)$.

\section{Discussion}

The territorial call of Dupont's larks results to be individually distinguishable by its spectrotemporal features. The complex nature of territorial calls, made up of several discrete types, each including up to five notes, allows a large number of individual combinations, and in turn permits measuring many spectrotemporal variables. In fact, the number of variables we measured was 3 to 10 times larger than that of previous studies on vocal individuality (Galeotti and Pavan 1991, Peake et al. 1998, Gilbert et al. 2002), and this determines the high classification success $(100 \%)$ of discriminant function analysis. Most studies on vocal individuality in birds were carried out on non-passerine birds with a simple repertoire (often made up of one note only). The fact that Dupont's lark belongs to the Alaudidae family, a group of birds well known for the complex, diverse and versatile repertoire, permits to reach finer level of vocal discrimination.

Voice recognition and capture-recapture data provided the same results in terms of territory switches and dispersal distances, both highlighting high territory fidelity and short distances moved by Dupont's lark males. The effectiveness of studies that rely on playback to attract birds has been debated, in relation to the possibility that playback can displace individuals from their original territories (Legare et al. 1999). This comparative study brings no evidence that playback causes territorial males to move off their territories, although it may cause short withinterritory displacements. Although this could be problematic in fine-tuned studies of habitat use, the short distance displacements potentially induced by the method are unlikely to bias crucial regional or landscape processes such as dispersal movements, and this method therefore appears adequate for the study of dispersal of elusive species. At the long term, we do not exclude that the effectiveness of playback to

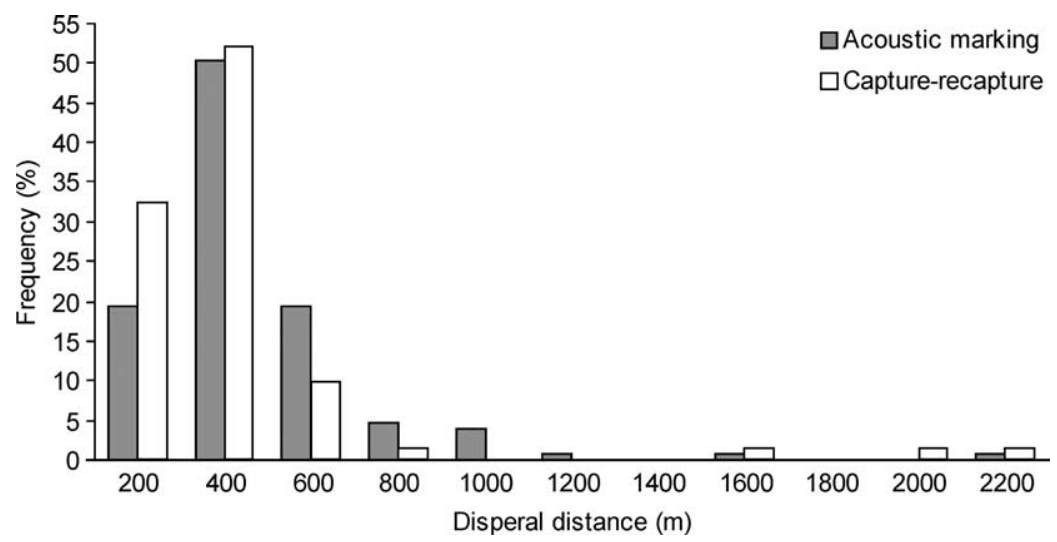

Fig. 4. Distribution of dispersal distance frequencies as resulted from acoustic marking and capture-recapture methods. 


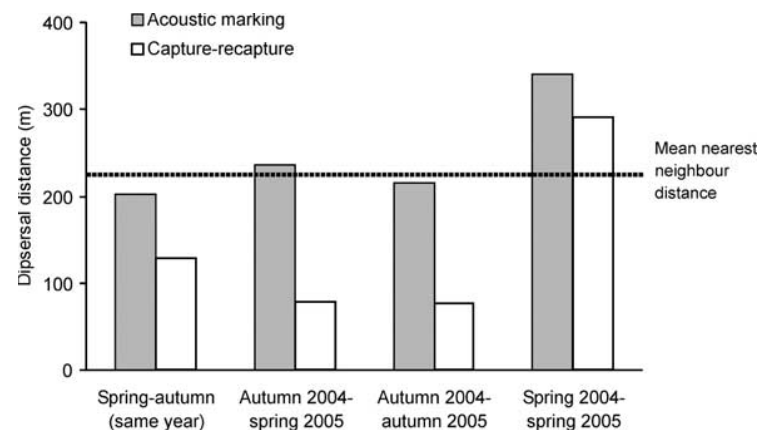

Fig. 5. Mean dispersal distance as resulted from acoustic marking and capture-recapture methods. The dotted line represents mean nearest neighbour distance.

attract individuals decreases with time due to individual habituation, or even that birds partially avoid playback areas after being trapped repeatedly in their surroundings.

The greatest difference among voice recognition and physical capture-recapture methods resides in relocation rate, which is significantly greater in the case of acoustic data. In all time periods, the proportion of relocations is significantly greater for acoustic data, but differences are marked above all in autumn. At this time of the year, most captures involve unmarked individuals, and re-capture success is scarce (8.9\%) compared to autumn acoustic data (41.5\%) or spring re-capture rate $(36.6 \%)$. We believe that captures in autumn mostly involve young floaters attempting to establish their first territory, although we were unable to distinguish them on morphological basis in the field. In the post-breeding period, up to 4 males can be captured in a single site in a day (pers. obs.). These floating individuals might gather to playback area using broadcasted songs as cues of conspecific presence. The occurrence of conspecifics would signal good habitat quality, especially in circumstances in which birds have scarce opportunity to sample it directly (Stamps 1988, Muller 1998, Danchin et al. 2004, Serrano et al. 2004) or lack personal information about it (Serrano et al. 2003). Nocera et al. (2006) showed that settlement decision of first-time breeders of bobolink Dolichonix oryzivorus was influenced by autumnal location cues such as decoys and playbacks, whereas mature males were not conditioned by it. Song playbacks have been shown to promote habitat occupancy also in the case of black-capped vireos Vireo atricapilla, and young birds were more responsive than older birds to conspecific vocalisations (Ward and Schlossberg 2004).

It is worth noting that $8-22 \%$ of all the individuals recorded in summer-autumn uttered calls presenting quavering harmonic structure and amorphous notes, and showed instability in call repertoire. These individuals were calling in the proximity of other males, and their presence in a site was not constant through time. We supposed these individuals were yearlings without permanent territories, which might develop their calls through a series of stages in which call quality improves gradually (DeWolfe and Baptista 1995). In spite of this, juveniles from the earliest spring broods can already utter stable territorial calls and occupy a territory in autumn: a young we caught and banded in spring 2004 was seen and recorded in autumn 2004, spring and autumn 2005, and showed stereotyped and stable calls in each recording session. Thus, depending on autumn recording date, young hatching date, motivation and probably other phenotypic characteristics, call repertoire of young individuals can be either stabilized or developing (DeWolfe et al. 1989). We did not included in voice recognition analyses males with unstable calls, because of difficulties in defining their call repertoires, but these might well enter in the sample of captured birds. Therefore, differences among voice recognition and capture-recapture methods might depend on the fact that they draw on slightly different sample of Dupont's lark males. Capture-recapture data are probably based on both floating and territorial males, whereas voice recognition mostly addresses to males that already acquired a territory.

Our results show that bioacoustics techniques can provide reliable information for studies in which the recognition of individuals is necessary. As voice recognition does not require individuals to be caught and banded, it has great logistic and welfare advantages. In our case, it also appears to be less time consuming than physic capture-recapture methods when considering field work, also taking into account that the greatest effort was initial, to prove acoustic stability over time within marked individuals that had to be recorded while sighted. As daily vocal activities are restricted to two brief periods (before dawn and at sunset), less time per day can be allocated in recording than in capturing, although the number of days of field work can be equal. Voice recognition method requires time to be spent in measurements, even if this activity is not constrained by season and time of day like field recording or capture sessions. Moreover, once proved individual acoustic constancy, measurements phase could be considerably shortened, as only one call train per individual could be measured to characterize its repertoire. In estimating method efficiency in the case of the Dupont's lark, it should be stressed that the prevalence of songs over calls can limit recording large daily samples of calls in the breeding period. Eventually, voice recognition is scarcely effective if young males are recorded in call development stage, and it is useless for monitoring females, which do not appear to utter longrange vocalisations. Although captures are male-biased, the capture-recapture method does permit to study Dupont's lark females (24\% of overall captures). 
All in all, the two methods produce similar results in terms of site fidelity and dispersal movements, whereas they differ in the case of re-location rates, possibly as a consequence of differences in the territorial status of target males. Such mismatch cannot be ignored, given that the estimates of many life-history parameters such as survival strongly depend on re-location rates. Capture-recapture method could provide more complete information on the population of young floaters. There is reason to suspect that a large proportion of male floaters occurs in Dupont's lark populations, because of highly male-biased adult sex-ratio (Tella et al. 2004). Although most ecological studies on birds are based on the territorial fraction of a population, it has been shown that monitoring silent floating individuals may permit a deeper knowledge of the future breeding population and increase the effectiveness of conservation efforts (Rohner 1996, Klomp and Furness 1992, Durell and Clarke 2004, Penteriani et al. 2005).

All things considered, the proportion of a population that is vocally active and responsive to playbacks might depend upon several factors, such as age, sex, time of the year, breeding and territorial status (Terry et al. 2005). These factors condition the performances of the two methods, and must be taken into account at the time of data interpretation. We suggest considering the two methods as complementary rather than alternatives for monitoring populations. Each method may offer unique information, and the two sources should be combined to provide correction factors that would eventually sharpen our knowledge of bird population ecology.

Acknowledgements - We wish to thank all the people that helped us during fieldwork: M. A. Carrero, J. M. Terrero, A. Mestre, E. Serrano, R. Imstepf and L. Bortolotti. We are also grateful to M. Méndez for sexing birds with molecular techniques, and to D. Blumstein and F. Sergio for providing useful comments. P. L. was supported by a Marie Curie IntraEuropean Fellowship within the 6th European Framework Programme, M. V. by an I3P pre-doctoral fellowship and D. S. by an I3P post-doctoral contract from the Consejo Superior de Investigaciones Científicas (Spain). Our methodology complies with the current Spanish laws in matter of avifauna capture.

\section{References}

Castelli, P. M. and Trost, R. E. 1996. Neck bands reduce survival of Canada geese in New Jersey. - J. Wildl. Manage. 60: 891-898.

Cramp, S. 1988. The Birds of the Western Palearctic. Vol. 5. - Oxford University Press, New York.

Danchin, E., Giraldeau, L. A., Valone, T. J. and Wagner, R. H. 2004. Public information: from nosy neighbours to cultural evolution. - Science 305: 487-491.
De Villiers, M. S., Meltzer, D. G. A., van Heerden, J., Mills, M. G. L., Richardson, P. R. K. and van Jaarsveld, A. S. 1995. Handling-induced stress and mortalities in African wildogs (Lycaon pictus). - Proc. R. Soc. B 262: 215-220.

DeWolfe, B. B. and Baptista, L. F. 1995. Singing behavior, song types on their wintering grounds and the question of leap-frog migration in Puget Sound White-crowned Sparrows. - Condor 97: 376-389.

DeWolfe, B. B., Baptista, L. F. and Petrinovich, L. 1989. Song development and territory establishment in Nuttal's white-crowned sparrows. - Condor 91: 397-407.

Durell, S. E. A. V. dit and Clarke, R. T. 2004. The buffer effect of non-breeding birds and the timing of farmland bird declines, Biol. Conserv. 120: 375-382

Eakle, W. L., Mannan, R. W. and Grubb, T. G. 1989. Identification of individual breeding bald eagles by voice analysis. - J. Wildl. Manage. 53: 450-455.

Galeotti, P. and Pavan, G. 1991. Individual recognition in male tawny owls (Strix aluco) using spectrograms of their territorial calls. - Ethol. Ecol. Evol. 3: 113-126.

Galeotti, P. and Sacchi, R. 2001. Turnover of territorial scops owls Otus scops as estimated by spectrographic analyses of male hoots. - J. Avian Biol. 32: 256-262.

Garza, V. and Suárez, F. 1990. Distribución, población y selección de hábitat de la Alondra de Dupont, Chersophilus duponti, en la Península Ibérica. - Ardeola 37: 312.

Garza, V., Suárez, F. and Tella, J. L. 2004. Alondra de Dupont Chersophilus duponti. - In: Madroño, A., González, C. and Atienza, J. C. (eds). Libro Rojo de las aves de España. Dirección General de Conservación de la Naturaleza-SEO/Birdlife, Madrid, pp. 309-312.

Garza, V., Traba, J. and Suárez, F. 2003. Is the European population of Dupont's lark Chersophilus duponti adequately estimated? - Bird Study 50: 309-311.

Gilbert, G., Tyler, G. A. and Smith, K. W. 2002. Local annual survival of booming male Great Bittern Botaurus stellaris in Britain, in the period 1990-1999. - Ibis 144: 51-61.

Kendall, W. L. and Nichols, J. D. 1995. On the use of secondary capture-recapture samples to estimate temporary-emigration and breeding proportions. - J. Appl. Stat. 22: 751-762.

Klomp, N. I. and Furness, R. W. 1992. Nonbreeders as a buffer against environmental-stress-Declines in numbers of great skuas on Foula, Shetland, and prediction of future recruitment. - J. Appl. Ecol. 29: 341-348.

Laiolo, P. and Tella, J. L. 2005. Habitat fragmentation affects culture transmission: patterns of song matching in Dupont's lark. - J. Appl. Ecol. 42: 1183-1193.

Laiolo, P. and Tella, J. L. 2006. Landscape Bioacoustics allows detection of the effects of habitat patchiness on population structure. - Ecology 87: 1203-1214.

Laiolo, P., Vögeli, M., Serrano, D. and Tella, J. L. 2005. Two new calls from the Dupont's lark Chersophilus duponti and imitations by other Alaudids: ecological and monitoring implications. - Ardeola 52: 167-172.

Legare, M. L., Eddleman, W. R., Buckley, P. A. and Kelly, C. 1999. The effectiveness of tape playback in estimating black rail density. - J. Wildl. Manage. 63: 116-125. 
Muller, K. L. 1998. The role of conspecifics in habitat settlement in a territorial grasshopper. - Anim. Behav. 56: 479-485.

Nocera, J. J., Forbes, G. J. and Giraldeau, L. A. 2006. Inadvertent social information in breeding site selection of natal dispersing birds. - Proc. R. Soc. B 273: 349-355.

Pasinelli, G., Schiegg, K. and Walters, J. R. 2004. Genetic and environmental influences on natal dispersal distance in a resident bird species. - Am. Nat. 164: 660-669.

Peake, T. M., McGregor, P. K., Smith, K. W., Tyler, G., Gilbert, G. and Green, R. E. 1998. Individuality in Corn Crake Crex crex vocalizations. - Ibis 140: 120-128.

Penteriani, V., Otalora, F., Sergio, F. and Ferrer, M. 2005. Environmental stochasticity in dispersal areas can explain the 'mysterious' disappearance of breeding populations. - Proc. R. Soc. B 272: 1265-1269.

Pradel, R. 1993. Flexibility in survival analysis from recapture data: handling trap-dependence. - In: Lebreton, J. D. and North, P. M. (eds). Marking individuals in the study of bird populations. Birkhäuser, Basel, Boston, Berlin, pp. 29-37.

Puglisi, L. and Adamo, C. 2004. Discrimination of individual voices in male great bitterns (Botaurus stellaris) in Italy. - Auk 121: 541-547.

Rohner, C. 1996. The numerical response of great horned owls to the snowshoe hare cycle: consequences of nonterritorial 'floaters' on demography. - J. Anim. Ecol. 65: 359-370.

Seoane, J., Justribó, J., Garcia, F., Retamar, J., Raban, C. and Atienza, J. C. 2006. Habitat-suitability modelling to assess the effects of land-use changes on Dupont's lark Chersophilus duponti: a case study in the Layna important bird area. - Biol. Conserv. 128: 241-252.
Serrano, D., Forero, M. G., Donazar, J. A. and Tella, J. L. 2004. Dispersal and social attraction affect colony selection and dynamics of Lesser kestrels. - Ecology 85: 34383447.

Serrano, D., Tella, J. L., Donázar, J. A. and Pomarol, M. 2003. Social and individual features affecting natal dispersal in the colonial lesser kestrel. - Ecology 84: 3044-3054.

Specht, R. 2003.Avisoft-SASLabPro. Sound Analysis and Synthesis Laboratory, version 4.23e. - Avisoft Bioacoustics, Berlin.

Stamps, J. A. 1988. Conspecific attraction and aggregation in territorial species. - Am. Nat. 131: 329-347.

Svensson, L. 1992. Identification guide to European passerines. 4th edition. Stockholm. 368 pp.

Tella, J. L., Carrete, M., Serrano, D. and Vögeli, M. 2004. High male-biased sex-ratios in breeding populations of steppe passerines: demographic and conservation implications. - In: Brotons, L. and Bota, G. (eds). Int. Symp. Ecol. Conserv. of Steppe-land birds. Lleida, Spain.

Tella, J. L., Vögeli, M., Serrano, D. and Carrete, M. 2005. Current status of the endangered Dupont's lark in Spain: overestimation, decline, and extinction of local populations. - Oryx 39: 90-94.

Terry, A. M. R. and McGregor, P. K. 2002. Census and monitoring based on individually identifiable vocalizations: the role of neural network. - Anim. Conserv. 5: 103-111.

Terry, A. M. R., Peake, T. M. and McGregor, P. K. 2005. The role of vocal individuality in conservation. - Front. Zool. 2: 10 .

Ward, M. P. and Schlossberg, S. 2004. Conspecific attraction and the conservation of territorial songbirds. - Conserv. Biol. 18: 519-525. 